Military Technical College

Kobry El-Kobbah

Cairo, Egypt

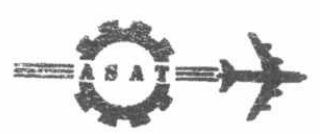

$10^{\text {th }}$ International Conference On Aerospace Sciences\&

Aviation Technology

\title{
OPTIMIZATION OF MIS/IL SOLAR CELLS PARAMETERS USING GENETIC ALGORITHM
}

Ahmed* K.A., Motaz“* M.S., Mohamed ${ }^{* * *}$ E.A. and Alaa S.H.

\section{ABSTRACT}

This paper presents a genetic algorithm optimization for MIS/IL solar cell parameters including doping concentration $\mathrm{N}_{\mathrm{A}}$, metal work function $\phi_{m}$, oxide thickness $d_{o x}$, mobile charge density $N_{m}$, fixed oxide charge density $N_{0 x}$ and the external back bias applied to the inversion grid $V$. The optimization results are compared with theoretical optimization and shows that the genetic algorithm can be used for determining the optimum parameters of the cell.

\section{KEY WORDS}

Photovoltaic, Solar cells, MIS/IL, Optimization and Genetic Algorithm.

\footnotetext{
* Professor, Dpt. of Electrical Engineering, Alexandria University, Alexandria, Egypt.

** Associate professor, Dpt. of Material Science, Alexandria University, Alexandria, Egypt. *** PhD., Air Defense Research Center, Cairo, Egypt

${ }^{\star * * *}$ Graduate Student, Dpt. of Radar, Air Defense College, Alexandria, Egypt.
} 


\section{INTRODUCTION}

Aluminum/thin SiOx/P-Si structure with an induced inversion layer MIS/L has been studied [1-4]. Its distinctive features are formed by shallow n-p junctions of about $0.1 \mu \mathrm{m}$ thickness arising under the influerice of a positive fixed charge in the insulating layer. However, practical achievement of high efficiency of MIS/IL solar cells was complicated by the presence of significant loss of generated power in the high-resistance inversion layer (IL). Some authors [5-7] have reduced the IL specific resistance by an increased of the built-in charge density and this made the MIS/IL technology complex and increase the cost of the MIS/IL solar cell. Several different improved methods were proposed. Among them Ref.[8], Built-in positive charge in the insulating layer was used for improving the conductivity of inversion layer of the underlying semiconductor surface. In addition, the difference of work functions between the semiconductor and the thin metal strips of the inversion grid was used. The inversion metal grid with a low work function was formed on top of an insulating layer of tunneling thickness between the strips of the collecting grid as shown in Fig.1. The aim of the work is to optimize the silicon MIS/IL solar cell parameters. The MIS model is based on the use of internal factors such as built-in charge including fixed oxide charge, mobile charge, interface trapped charge, doping concentration, oxide thickness and metal work function. External parameters will be included namely, external back bias on the inversion grid [9].

\section{MODELLING OF MIS/IL SOLAR CELLS}

The electronic energy band diagram of MIS/IL is shown in Fig.2. The dependence of of surface potential $\Psi_{\mathrm{s}}$ on the vaiue of external back bias $V$ of the inversion grid is calculated from the equation of charge neutrality [9],

$$
\begin{aligned}
\frac{d}{\varepsilon_{o x}} \sqrt{2 k T \varepsilon_{s} N_{A}} * F( & \left(\beta \psi, \frac{n_{p o}}{p_{p o}}\right)+q N_{m}+q N_{a x}-q^{2} D_{i t}\left(\psi_{s}+v_{p}+\frac{E_{c}}{q}\right)= \\
& \mathrm{E}_{\mathrm{g}}-\phi_{m}+\chi_{s}-\psi_{s}-v_{p}+V
\end{aligned}
$$

where

$$
F\left(\beta \psi, \frac{n_{p o}}{p_{p o}}\right)=\left(\left(e^{-\beta \psi}+\beta \psi-1\right)-\frac{n_{p o}}{p_{p o}}\left(e^{\beta \psi}-\beta \psi-1\right)\right)^{0.5}
$$

$n_{p o}, p_{p o}$ are the equilibrium densities of electrons and holes respectively, $\beta=q / k T, d_{o x}$ is the thickness of the interfacial layer, $D_{\text {it }}$ is the interface traps density (states $/ \mathrm{cm}^{2}$ $\mathrm{eV}$ ), $\quad \mathrm{V}$ is the external back bias applied to the inversion grid, $\phi_{\mathrm{m}}$ is the metal work function, $\chi_{s}$ is the electron affinity of the semiconductor and $E_{g}$ is the energy gap of the semiconductor.

The potential barrier height of the MIS/IL structure $\phi_{\mathrm{bp}}$ is the sum of the band bending and the value of Fermi level $V_{p}$ in the bulk as shown in Fig. 2. 


$$
\phi_{b p}=\psi_{s}+v_{p}=\psi_{s}+\frac{k T}{q} \ln \left(\frac{N_{v}}{N_{A}}\right)
$$

Where $N_{v}$ is the effective density of states in the valence band. Therefore, the back bias increase the value of $\phi_{\mathrm{bp}}$. The potential barrier height is related to the saturation current density $J_{s}$ of the MIS/LL structure by[10],

$$
J_{s}=A^{* * *} T^{2} e^{\left(\frac{q \phi_{\phi_{p}}}{k T}\right)} e^{\left(\left(-q \phi_{T}{ }^{0.5} d\right)\right.}
$$

Where $A^{* *}$ is the effective Richardson constant and $q \phi_{T}$ is an average barrier height in the insulating layer with thickness $d$. The dependence of $x$ on the potential $\Psi[7]$ is given by,

$$
x=\left(\frac{\varepsilon_{s}}{2 k T N_{A}}\right)^{\frac{1}{2}} \int_{\psi(x)}^{\psi}\left(\beta \psi+\frac{n_{p o}}{p_{p o}} e^{\beta \psi}\right)^{\frac{1}{2}} d \psi
$$

The thickness $t$ of the IL by calculating of the cross point of $\Psi(x)$ with the Fermi level. The value of $\Psi_{b}$ is taken as the lower bound of the integral in the equation (4), so we get,

$$
t=\left(\frac{\varepsilon_{s}}{2 k T N_{A}}\right)^{\frac{1}{2} \psi_{s}} \int_{\psi_{b}}\left(\beta \psi+\frac{n_{p o}}{p_{p o}} e^{\beta \psi}\right)^{\frac{1}{2}} d \psi
$$

The MIS/LL solar cell photocurrent can be represented as the sum of the electron and hole currents, generated by the light flux $F$ in the field of the base and induced IL [9] and is given by,

$$
J_{L}=q F(1-R) \alpha\left(\frac{L_{1} v_{n} e^{v_{n} t}+L_{2} v_{n} e^{-v_{n} t}+\alpha e^{-\alpha t}}{\alpha^{2}-v_{n}^{2}}+\frac{K_{1} v_{p} e^{v_{p} t}+K_{2} v_{p} e^{-v_{p} t}+\alpha e^{-\alpha t}}{\alpha^{2}-v_{p}^{2}}\right)
$$

where

$$
\begin{aligned}
& L_{1}=\frac{e^{-\left(v_{n} t+\alpha\right)}-e^{-\left(\alpha+v_{n} l\right)}}{2 \sinh \left(v_{n} l-v_{n} t\right)} \\
& L_{2}=\frac{e^{\left(v_{n} t+\alpha\right)}-e^{-\left(\alpha+v_{n} l\right)}}{2 \sinh \left(v_{n} l-v_{n} t\right)} \\
& K_{1}=\frac{(\alpha+h) e^{-v_{p} t}-\left(v_{p}-h\right) e^{-\alpha}}{\left(v_{p}+h\right) e^{v_{p} t}-\left(v_{p}-h\right) e^{v_{p} t}} \\
& K_{2}=\frac{(\alpha+h) e^{v_{p} t}+\left(v_{p}-h\right) e^{-\alpha t}}{\left(v_{p}+h\right) e^{v_{p} t}-\left(v_{p}-h\right) e^{-v_{p} t}}
\end{aligned}
$$




$$
h=\frac{S}{D_{p}}
$$

$V_{n}$ and $V_{p}$ are are the inverse to the diffusion length of electrons and holes, $S$ is the surface recombination velocity on the frontal surface of the solar cell, $D_{p}$ is the hole diffusion coefficient, $R$ takes into account the part of the photon flux reflected from the surface of the substrate with thickness $\mid$ and $\alpha$ is the optical absorption coefficient. The MIS/IL solar cell parameters will optimized by maximizing the efficiency using genetic algorithm given that [11],

$$
\eta=\frac{I_{s c} \times V_{o c} \times F F}{P_{i n}}
$$

where

$$
F F=\frac{V_{m} I_{m}}{V_{o c} I_{s c}}
$$

$V_{m}$ and $I_{m}$ are the voltage and current corresponding to the maximum power, respectively, FF is the fill factor and $\mathrm{P}_{\text {in }}$ is the power of the light for the AMO spectrum $\left(135.3 \mathrm{~mW} / \mathrm{cm}^{2}\right)$.

\section{3- OPTIMIZATION PROCESS}

Genetic algorithm has been used to optimize the MIS/IL solar cell parameters by changing the device physical parameters namely, doping concentration $\mathrm{N}_{\mathrm{A}}$, oxide thickness $d_{0 x}$ metal work function $\phi_{m}$, external back bias on the inversion grid $V$, mobile charge density $N_{m}$ and fixed oxide charge density $N_{f}$ [12]. Binary encoding scheme is used in this algorithm to encode the MIS/LL solar cell parameter [13-14]. The chromosome contains all parameters as shown in Fig.3. Each gene parameter encoded as 4-bit to include sixteen quantized values, as shown in Fig.4, and eight populations are selected to be greater than the number of genes per chromosome. Elitism is used to save the best solutions to improve the performance of the genetic algorithm [14].

The algorithm is started with a set of solutions (represented by chromosomes) called population. Solutions from one population are used to form a new population. This is motivated by a hope, that the new population will be better than the old one. Solutions which are selected to form new solutions (offspring) are selected according to their fitness. The more suitable they are the more chances they have to be reproduced. This is repeated until some conditions (for example number of populations or improvement of the best solution) is satisfied. The genetic algorithm proceed as follows, [13]

(1) Create a population of random individuals which represents a possible solution to the problem at hand.

(2) Evaluate each individual fitness i.e. its ability to solve the specified problems.

(3) Select individual population members to be parents. 
(4) Produce children by recombining parent's material via crossover and mutation and add them to the population.

(5) Evaluate the children fitness.

(6) Repeat steps (3-5) until a solution with the desired fitness goal is obtained [15-16]. The genetic algorithm flow chart of the optimization problem is shown in Fig.5.

\section{Optimization results:}

The optimization process proceeds to obtain the maximum efficiency of the MIS/LL solar cell by optimizing the cell parameters. The optimization process depends on the number of iterations needed to achieve the maximum efficiency of the cell. After 40 iterations (Fig.6), the efficiency reaches a maximum steady state value of $22.95 \%$. Table 1. shows the parameters required to achieve the this efficiency (data(1)). The tabie also includes the results of the optimization process by using aluminum as a contact metal $\left(\phi_{m}=4.1\right)$. In this case, only five parameters are used for optimization (data(2)). The results of the optimization process when using aluminum as metal contact and with no external back bias $(\mathrm{V}=0)$ (is shown in data (3)).

The optimization results are compared with a theoretical optimization based on an analysis of structure parameters [7]. The comparison has shown good agreement of the genetic algorithm optimization with the other mentioned method as shown in Table 2 . 


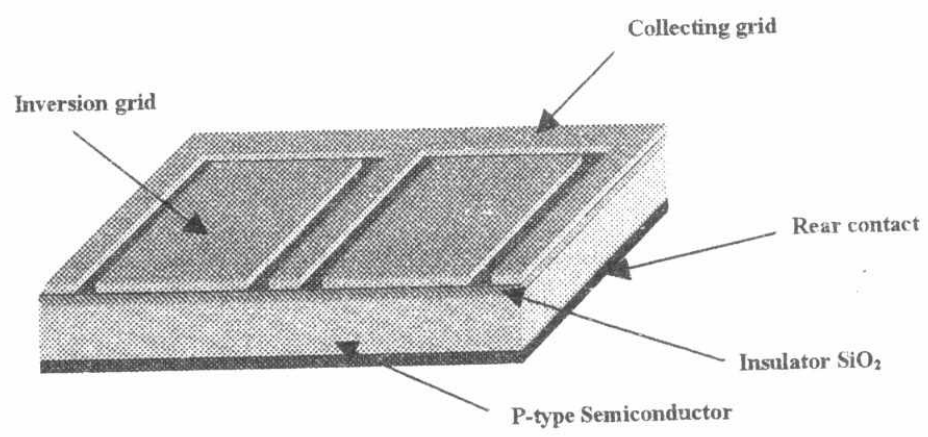

Fig.1. Schematic diagram of MIS/IL solar cell structure

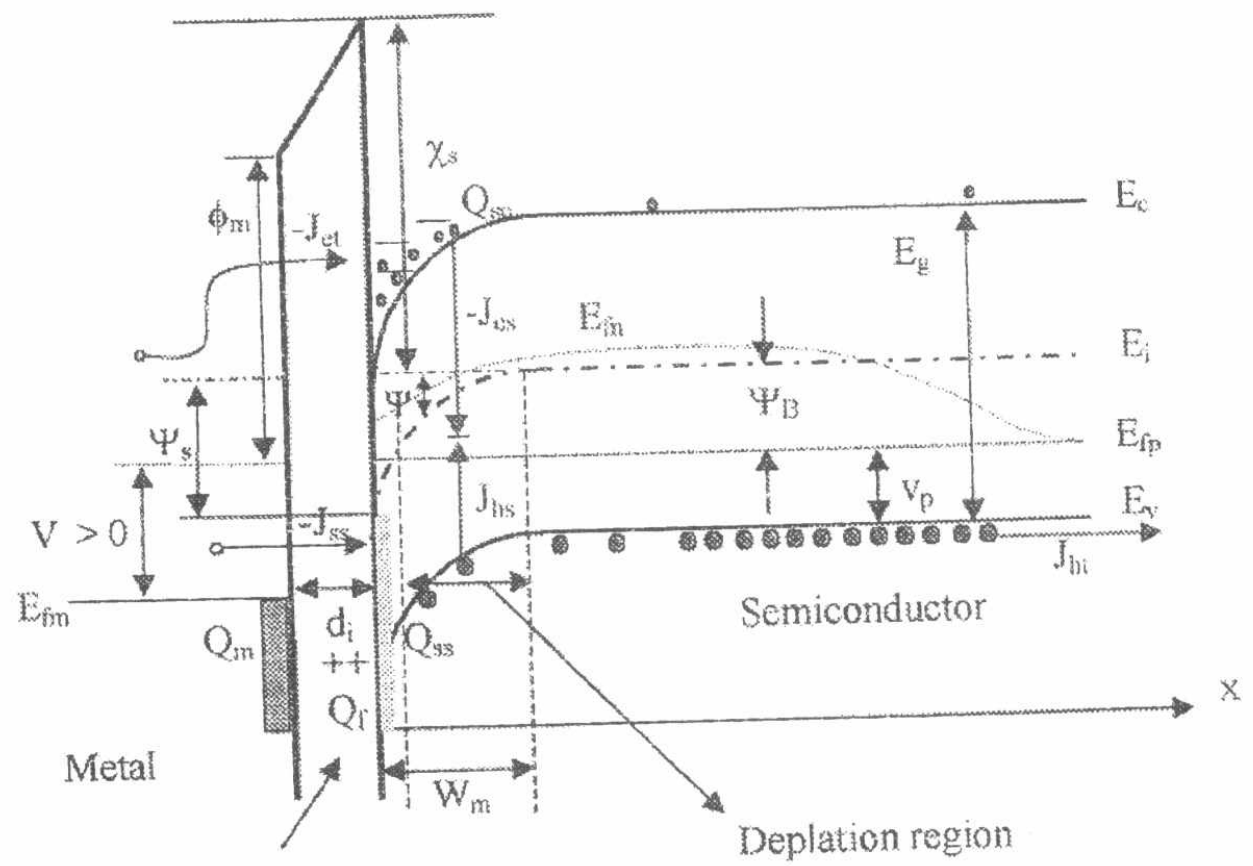

Insulator

Fig.2. Energy band diagram of MIS/IL Solar cell 

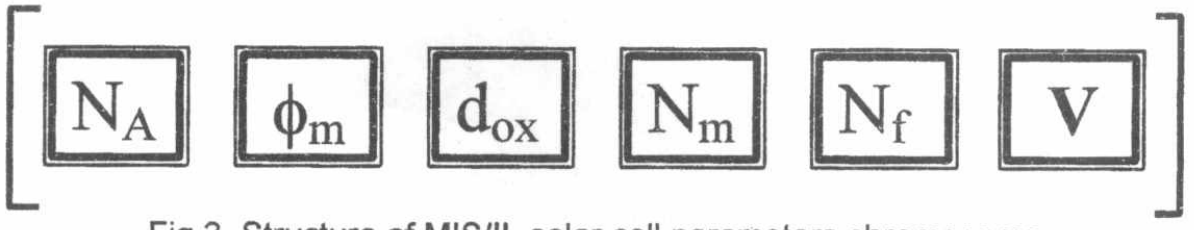

Fig.3. Structure of MIS/IL solar cell parameters chromosome

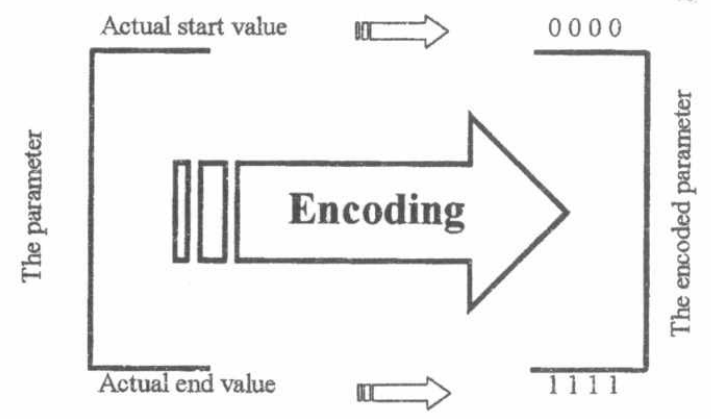

Fig.4. The binary encoding of the MIS/IL solar cell parameters

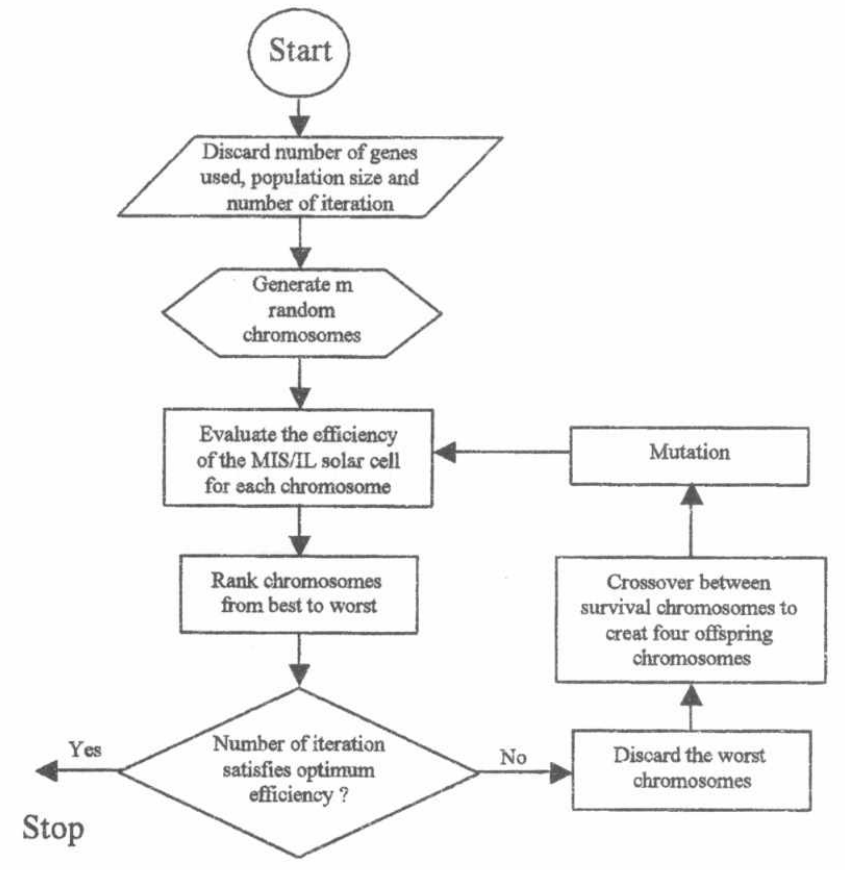

Fig.5. The flow chart of the genetic algorithm for MIS/IL solar cells 


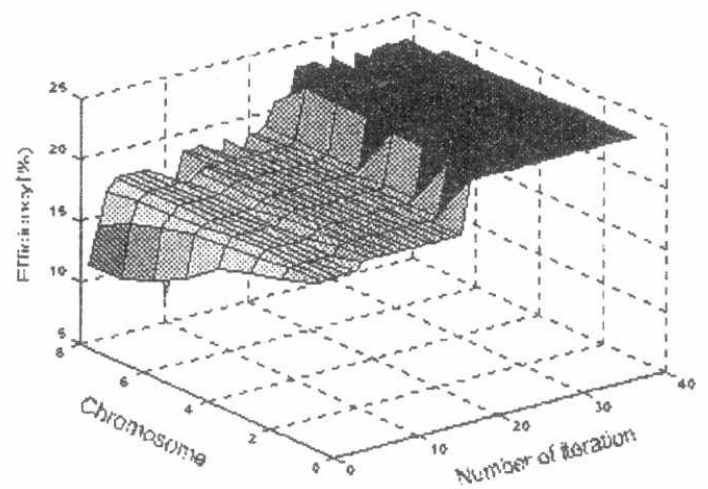

Fig.6. The optimization process during 40 iterations.

Table 1. Parameters required to achieve the optimum efficiency

\begin{tabular}{|c|c|c|c|c|c|c|c|}
\hline $\begin{array}{l}\text { Optimization } \\
\text { results }\end{array}$ & $\begin{array}{c}\mathrm{N}_{\mathrm{A}} \\
\left(\mathrm{cm}^{-3}\right)\end{array}$ & $\begin{array}{c}\phi_{\infty} \\
(e V)\end{array}$ & $\begin{array}{l}\mathrm{d}_{\mathrm{os}} \\
\left(\mathrm{A}^{\circ}\right)\end{array}$ & $\begin{array}{c}\mathrm{N}_{+} \\
\left(\mathrm{cm}^{-2}\right)\end{array}$ & $\begin{array}{c}\mathrm{N}_{f} \\
\left(\mathrm{~cm}^{-2}\right)\end{array}$ & $\begin{array}{l}V \\
(y)\end{array}$ & $\begin{array}{l}\text { Optimum } \\
\text { efficiency (\%) }\end{array}$ \\
\hline Encoded data (1) & 0110 & 0100 & 0011 & 1111 & 0100 & 1110 & \multirow{2}{*}{22.95} \\
\hline Decoded data (1) & $3 \times 10^{16}$ & 3.4 & 20 & $5 \times 10^{13}$ & $3 \times 10^{11}$ & 0.7 & \\
\hline Encoded data (2) & 0110 & 1011 & 0011 & 1111 & 1010 & 1111 & \multirow{2}{*}{22.5} \\
\hline Decoded data (2) & $3 \times 10^{16}$ & 4.1 & 20 & $5 \times 10^{13}$ & $5 \times 10^{12}$ & 0.75 & \\
\hline Encoded data (3) & 0110 & 1011 & 0011 & 1111 & 0100 & 0000 & \multirow{2}{*}{21.95} \\
\hline Decoded data (3) & $3 \times 10^{16}$ & 4.1 & 20 & $5 \times 10^{13}$ & $5 \times 10^{12}$ & 8 & \\
\hline
\end{tabular}

Table 2. The comparison between three optimization process

\begin{tabular}{|c|c|c|}
\hline The model & Present model & $\begin{array}{c}\text { Theoretical model } \\
{[7]}\end{array}$ \\
\hline Method used & Genetic algorithm & $\begin{array}{c}\text { Analysis of structural } \\
\text { parameters }\end{array}$ \\
\hline$V_{\propto}[\mathrm{m}$ V] & 735 & 715 \\
\hline $\mathrm{J}_{\propto}[\mathrm{mA} / \mathrm{cm}]$ & 46.1 & 42 \\
\hline$\Psi_{,}[\mathrm{mV}]$ & 1060 & 1050 \\
\hline FF $[\%]$ & 82 & 81 \\
\hline Efficiency [\%] & 22.95 & 20.5 \\
\hline
\end{tabular}




\section{References}

[1] Dahl-Kwang Yeh and Thomas A. Demassa "Computer analysis of Induced-Inversion Layer MOS solar cells", Solid State Electronics, Vol. 27, No. 3, pp. 283-292, March 1984.

[2] M.Y.Deghish and F.D.Ho "A comprehensive analysis model for MIS devices", IEEE, Tranaction on Electron Devices, Vol. 39, No. 12, pp. 2771-2780, December 1992.

[3] C.R.Fang and J.R "Comparison of theoretical and experimental solar cell performance", 13th IEEE Photovoltaic Specialists Conference, pp 1318-1326, December 1978.

[4] C.E.Norman and R.E. Thomas "Detailed modeling of inversion layer solar cells", IEEE, Transaction on Electron Devices, ED-27, pp. 731-737, 1980.

[5] H.Nenhaus.B.Kuhlman, R.Meyer. Ametz.R.Auer, A.G.Aberle, and R.Hezel "Determination of the dominant current mechanism in the MIS tunnel diode of MIS Inversion-Layer silicon solar cells", 2th World conference And Exhibition on Photovoltaic Solar Energy Conversion, Vienna, July, 1998.

[6] A.A.Abdou and S.E.D Habib "Optically induced inversion in MIS solar cell", Solid State Electronics, Vol. 29, No.8, pp. 751-758, August 1986.

[7] V. Yu. Yerokhov, I. I. Melnyk and A.V. Korovin "External bias as the factor of efficiency increase of silicon MIS/IL solar cells", Solar Energy and Solar cells, ED-58, pp. 225-236, 1999.

[8] Burkhard Kuhlman, Armin G.Aberle, Rudolf Hezel, and Gernot Heiser "Simulation and optimization of Metal-Insulator-Semiconductor Inversion-Layer silicon solar cells", IEEE Transaction Electron Devices., Vol. 47, No. 11, pp. 2167-2178, 2000.

[9] Ahmed.K.Aboul-soud, Neama Elfaramawy, Mohamed.E.Attala and Alaa.S.Hafez "Hieg effeiency silicon MIS/IL solar cells under external back bias" National radio science conference, NRCS'2002, Alex, March,19-21,2002 pp527-534.

[10] S.M.Sze "Physics of semiconductor devices", John Wiely, New York, 1981.

[11] M.B.Saleh, M.M.Soliman, M.E.Attalla, A.Hafez "Computer modeling for MIS/IL solar cells", $8^{\text {th }}$ International Conference, ICCT, Alexandria, September1998.

[12] M.B.Saleh, M.M.Soliman, M.E.Attalla, A.S.Hafez "Effects of metal properties on MIS/IL solar cells", $16^{\text {th }}$ National Radio Science Conference, NRSC'99, Cairo, February 1999.

[13] Zbigniew Michalewiez "Genetic algorithms + Data structure = Evolution programs", Springer, Veriag Berlin Heidelberg, New York, 1996.

[14] Internet site: hittp://cs.felk.cvut.cz/xobtiko/ga.com, "Genetic Algorithm", Nov. 2002.

[15] Randy L.Haupt "An introduction to genetic algorithm for electromagnetics", IEEE, Anennas and propagation magazine, Vol.37, No. 2, 1995.

[16] Alaa.S.Hafez"Msc thesis", "Optimization of MIS/IL solar cells efficiency using genetic algorithm",Alexandria University, April, 2002. 